www.pasosonline.org

\title{
Calidad en el sector turístico: marco normativo y planificación
}

\author{
Ma Remedios Zamora Roselló* \\ Universidad de Málaga (España)
}

\begin{abstract}
Resumen: En este artículo se analiza la calidad desde la perspectiva del sector público, incidiendo en la participación de la Administración General del Estado y de las Comunidades Autónomas, con especial atención a la regulación e instrumentos de planificación adoptados en Andalucía. La perspectiva autonómica se completa con el estudio de las iniciativas adoptadas en Cataluña y en Canarias; esta selección tiene su origen en la experiencia turística de estas Comunidades y el alto número de visitantes, nacionales e internacionales, que reciben anualmente. El examen de la evolución en las disposiciones y en los planes adoptados, permite conocer cuáles son los objetivos y principios para la consecución de la calidad que se están fomentando desde las Administraciones Públicas, así como el nivel de adaptación de estos fines a las demandas del sector privado y de la ciudadanía. La metodología empleada ha sido el análisis jurídico del marco normativo vigente, así como de los instrumentos de planificación adoptados, y la documentación con fuentes bibliográficas.
\end{abstract}

Palabras Clave: Administración pública; Sostenibilidad; Competitividad; Accesibilidad; Residentes y visitantes; Andalucía.

\section{Quality in the tourism sector: regulatory framework and planning}

Abstract: In this article we analyse quality from the perspective of the public sector, focusing on the participation of the General Authorities of the State Administration and Autonomous Communities in Spain, with special focus on the regulatory planning instruments adopted in Andalusia. This regional perspective is completed with the study of initiatives adopted in Catalonia and the Canary Islands. The selection is based ion the longstanding history of experience in tourism of these Autonomous Communities and the large numbers of visitors, both national and international, who are received by them annually. The study of the evolution of planning articles and laws is essential in order to understand the aims and principles of quality control as defined by the Public Authorities, and their adaptation to private sector demands and the general citizens. The methodology is based on legal analysis of the current normative framework, and the planning instruments adopted, together with other bibliographic sources.

Keywords: Public Authorities; Sustainability; Competitiveness; Accessibility; Residents and visitors; Andalusia.

\section{Introducción}

En un sector en constante evolución como es el turístico, la necesidad de alcanzar unos estándares de calidad y de mejorar continuamente en los niveles ofrecidos, se convierte en una premisa básica para que un destino pueda ser competitivo a nivel mundial. Las exigencias de los usuarios han evolucionado en los últimos años gracias a las posibilidades que le ofrecen la multitud de ofertas y servicios, y a las herramientas actuales para elaborar una comparativa adaptada a sus necesidades sobre calidad y precio. El potencial visitante elige su destino tras una búsqueda y selección en la que se priman las valoraciones y opiniones de otros viajeros, y la información del destino ofrecida por otros cauces, como la publicidad comercial o institucional.

Universidad de Málaga, España; E-mail: remedios@uma.es 
El Consejo Ejecutivo de la Organización Mundial del Turismo, en su $103^{\mathrm{a}}$ sesión celebrada en mayo de 2016 en la ciudad de Málaga, definió la calidad de un destino turístico como el "resultado de un proceso orientado a atender todas las necesidades de productos y servicios turísticos, los correspondientes requisitos y las expectativas del consumidor a un precio aceptable de conformidad con condiciones contractuales aceptadas mutuamente y con factores subyacentes implícitos como la seguridad, la higiene, la accesibilidad, la comunicación, la infraestructura y los servicios y comodidades públicos. También presenta aspectos relativos a la ética, la transparencia y el respeto del entorno humano, natural y cultural" (OMT, 2016).

En base a esta definición y a las demandas actuales de los turistas, se plantea qué pueden aportar las administraciones públicas en el ámbito de la calidad turística; cuáles son las herramientas que desde el sector público pueden ofrecerse para garantizar estos estándares de calidad y conseguir poner en valor los recursos de un entorno, garantizando su sostenibilidad y potenciando la competitividad de un destino. Estos son los interrogantes que desde hace décadas llevan tratando de responder los instrumentos de planificación turística que, a nivel estatal y autonómico, se han adoptado en nuestro país.

Por tanto, la calidad no es un concepto novedoso aplicado a la política turística ni a la planificación administrativa en esta materia, las novedades radican en cómo se están adaptando las Administraciones Públicas a las exigencias de un mercado en constante transformación, destinos consolidados y usuarios con un mayor conocimiento sobre el destino y más exigentes en sus demandas. A todo ello cabe añadir los desafíos que implica el turismo colaborativo, donde surgen nuevos modelos de negocio y servicios para el turista que escapan a las figuras tradicionales, por lo que desde la planificación y la regulación hay que ofrecer respuestas a esta evolución del sector turístico.

En esta consecución de un modelo de planificación administrativa que impulse la calidad en el sector turístico es clave la implicación del sector privado, como instrumento de ejecución efectiva de buena parte de las iniciativas auspiciadas desde el ámbito público. La correlación de responsabilidades entre las administraciones públicas y los empresarios y trabajadores de este ámbito constituyen la única vía posible para alcanzar un modelo turístico de calidad (Mariño Romero, 2013).

En los últimos años, la proliferación de los reconocimientos y la necesidad de homogeneizar y normalizar los criterios y estándares de calidad también están marcando las iniciativas públicas y privadas en este ámbito ${ }^{2}$. La integración de los distintivos de calidad en las políticas públicas exige de figuras que tengan una repercusión real sobre la opinión pública y permitan posicionar favorablemente al servicio o al establecimiento que ha alcanzado ese logro. Todo ello, sin olvidar la relevancia de la difusión de las experiencias que permiten las redes sociales; son las opiniones y valoraciones de los usuarios las que también condicionan la elección de un servicio turístico, e incluso la de un destino.

\section{Calidad turística y Administración General del Estado}

En el marco de sus competencias, desde la Administración General del Estado hace décadas que se lleva trabajando sobre la calidad en el sector turístico ${ }^{3}$. En este sentido, corresponde recordar que ya a finales del siglo pasado se adoptaron iniciativas como el Sistema de Calidad de Hoteles y Apartamentos, fomentando la implicación del sector privado en la mejora de la calidad de sus productos y servicios. Estas primeras iniciativas, adoptadas en el ámbito del Plan Marco de Competitividad del Turismo Español (FUTURES I y II), daban respuesta a las demandas de un nuevo modelo turístico en el que comenzaba a primar la calidad frente a la cantidad, caracterizado por un consumidor cada vez más exigente, y por una ciudadanía que planteaba nuevos retos para el control y eliminación de los impactos negativos del turismo como la degradación ambiental o la excesiva ocupación del suelo (Beas Secall, 2012).

El Plan de Calidad Turística Española supuso un punto de inflexión en la implantación de un sistema de gestión y mejora de la calidad de los productos y servicios turísticos; asimismo, desde la Administración General del Estado se ofrecieron los instrumentos institucionales para el desarrollo de una Marca de Calidad Turística Española, y se fomentó la participación de las organizaciones empresariales del sector turístico en los organismos internacionales de normalización (Navarro de Vega, 1999).

Siguiendo esta línea, el Plan Horizonte 2020-Plan 2008-2012, continuó potenciando la calidad como premisa para el desarrollo del sector. A lo largo del texto del Plan fueron numerosas la referencias a distintas perspectivas de la calidad; no obstante, consideramos de especial interés la consideración de la calidad como elemento de diferenciación en los mercados y de mejora en la gestión (Pérez Guerra, 2014). Desde el Plan se hacía una llamada a la "calidad experiencial", apostando por una mayor implicación de los empresarios turísticos y las administraciones turísticas locales, con el apoyo de la Administración 
General del Estado y de las distintas administraciones autonómicas. La finalidad era clara: que la calidad turística fuera un instrumento para añadir valor a los productos turísticos.

El Plan Nacional e Integral del Turismo 2012-2015, analiza la calidad como elemento diferenciador del "destino España" frente a la competencia que suponen otros destinos emergentes con ventajas respecto al precio de los servicios. El Sistema de Calidad Turístico Español desarrollado por la Administración General del Estado, y ya plenamente consolidado en esta etapa, tiene una clara finalidad: "dotar a las empresas y destinos de instrumentos contrastados de gestión interna óptima, que contribuyan a la reducción de sus costes operativos y a la mejora de la relación calidad/precio de los bienes y servicios que aquellas ofrecen a sus clientes" (Álvarez García, 2012).

A pesar de la ya mencionada consolidación de este Sistema Nacional de Calidad, las deficiencias detectadas en cuanto al nivel de percepción de la calidad por parte de los turistas, exige la adopción de nuevas medidas para adaptar las iniciativas a las demandas del mercado (Fuentes Medina, 2016). En este sentido, en el texto del Plan se apuesta por situar a los empresarios como actores determinantes del Sistema, configurando de esta forma un modelo de corresponsabilidad entre el sector público y el privado ${ }^{4}$.

\section{Normativa y planificación en la Comunidad Autónoma de Andalucía}

\subsection{Antecedentes normativos: la Ley $12 / 1999$}

La Ley 12/1999, del Turismo de Andalucía, abordaba la calidad desde distintas perspectivas. El análisis del articulado de esta disposición, nos permite comprobar como entre sus fines se enumeraba expresamente la protección de los recursos turísticos de acuerdo con el principio de sostenibilidad; la consecución de la competitividad del sector a partir de la incorporación de los criterios de calidad a la gestión de las empresas y servicios turísticos, incidiendo en la profesionalidad y cualificación del personal; y, por último, la protección de los usuarios turísticos (art. 1.2). En opinión de Sanz Domínguez, la calidad de los servicios turísticos era empleada en este texto como criterio de clasificación de los establecimientos turísticos; como un derecho del usuario turístico contrapuesto al correlativo deber del empresario; como instrumento de tipificación de determinadas infracciones administrativas, y como meta para conseguir un mayor nivel de profesionalización en el sector (Sanz Domínguez, 2003) .

En la misma línea, y entre los objetivos generales de la ordenación y promoción de los recursos turísticos se establecía un mandato claro a todas las Administraciones Públicas, para que estimularan "la mejora de la calidad y de la competitividad de la oferta turística andaluza respetando el entorno natural y cultural" (art. 14). Entre las acciones destinadas a la consecución de estos fines se incluían previsiones para la creación de infraestructuras técnicas y de servicios, la realización de estudios para la mejora de la competitividad, la modernización de los establecimientos, el apoyo a la mejora de la calidad de los establecimientos turísticos y a la formación de los profesionales del sector, y el desarrollo de actividades de promoción, creación y comercialización de productos turísticos, entre otros (Larrubia Vargas, 2001).

Asimismo, la norma andaluza también preveía incentivos a la calidad, mediante la creación y otorgamiento por parte de la Consejería competente de distintivos de calidad; además de la concesión de medallas, premios y galardones que permitieran reconocer y promocionar las actuaciones a favor del turismo (art. 21).

Desde el punto de vista organizativo, la Ley 12/1999 estableció las bases para la creación de la Oficina de la Calidad del Turismo; órgano de titularidad pública cuyo objetivo era velar por la efectividad y garantías de los derechos de los turistas, así como garantizar la calidad de los servicios turísticos prestados en la Comunidad (art. 12). Parte de la doctrina ha destacado la importancia de este precepto ya que suponía "la creación de un organismo público dedicado a velar en exclusiva por la calidad turística con las consecuencias, jurídicas y de gestión que ello conlleva. Dada la dimensión adquirida por la calidad, la especialización dela Administración en esta materia resulta obligada. No debería tratarse de meros órganos asesores. Deben tener una función asesora pero junto a ella también deben tener una función ejecutiva (inspectora, otorgamiento de distintivos) y una muy significativa función de investigación y formación" (Tudela Aranda, 2003: 145).

Las funciones principales de este órgano eran de investigación y análisis de todos los aspectos que inciden en la calidad del turismo, así como de la información sobre la prestación de los servicios turísticos; asimismo, también ostentaba competencias para formular propuestas a la Consejería sobre los criterios para la concesión de los distintivos de calidad. Estas competencias se concretaron a través 
de los Decretos de desarrollo de la Ley andaluza ${ }^{5}$, que también incluyeron entre las funciones de la Oficina la identificación de las necesidades de formación y capacitación de los profesionales del turismo en la calidad turística; el análisis de la implantación y uso de los sistemas de certificación de calidad y su relación con los sistemas de normalización; así como la formulación de propuestas para la adopción de medidas y acciones de difusión, promoción y fomento de la calidad turística, dirigidas tanto a las empresas turísticas como a los usuarios de servicios turísticos.

La composición de esta Oficina trataba de dar respuesta a la necesidad de integrar a todos los actores públicos y privados del sector turístico en el fomento de la calidad; de ahí que formaran parte de este organismo representantes de la administración autonómica, de la Federación de Municipios y Provincias de Andalucía, de las organizaciones empresariales y sindicales más representativas y del Consejo de los Consumidores y Usuarios de la Comunidad. El carácter interdisciplinar de la calidad queda de manifiesto a través de los representantes autonómicos de las distintas áreas implicadas: turismo, consumo, calidad de los servicios públicos, innovación, ciencia y empresa, empleo, cultura y medio ambiente.

\subsection{Antecedentes planificadores: el Plan de Calidad Turística de Andalucía 2006-2008}

La primera de las actuaciones dedicadas íntegramente a la calidad se concretó en la Comunidad Autónoma de Andalucía a través del Plan de Calidad Turística de Andalucía 2006-2008, con la finalidad última de garantizar el liderazgo de Andalucía como destino de calidad en el mercado turístico. Los cuatro ámbitos de actuación en los que se centraba el Plan de Calidad eran: el espacio turístico, las empresas, las personas y el producto turístico. Las propuestas sobre el espacio turístico se asentaban sobre la premisa de la calidad en destinos; por ello, se atendía a la relación entre las políticas en materia de turismo y de ordenación del territorio. Este contexto territorial se completaba con la necesidad de desarrollar estrategias de complementariedad entre los destinos turísticos andaluces, en la búsqueda de una oferta más diversificada. Esta apuesta por la complementariedad de los destinos también se ha mantenido en los vigentes instrumentos de planeamiento, como en el actual Plan General de Turismo Sostenible de Andalucía Horizonte 2020 (PGTS).

En estrecha relación con el contenido del PGTS se encuentra la mención a la sostenibilidad; elemento transversal que, en esta primera etapa en la ordenación de la calidad ya se incluía en esta planificación (Castro León, 2005). La eficacia en la consecución de estos fines se apoyaba en la cooperación interadministrativa, tratando de implicar a todos los niveles administrativos competentes, además de la indispensable colaboración del sector privado. La participación de trabajadores y empresarios para alcanzar los estándares de calidad era ya una prioridad reconocida en este primer Plan de Calidad.

La presencia de la calidad, como premisa en la actuación de las empresas, se centraba en la obtención de certificaciones y distinciones de calidad. A estas certificaciones se les reconoce una doble virtualidad, ya que incentivan la adopción de medidas de mejora por parte del sector privado; a la vez que mejoran la imagen de Andalucía como destino turístico, actuando como reclamo publicitario y atrayendo a los potenciales visitantes (Hernández Ramírez, 2008).

Los principios de calidad aplicados a las personas se traducían en una serie de objetivos de aplicación tanto al sector público como al sector privado. Era de interés esta propuesta por cuanto aplicaba los criterios de mejora en la formación para el personal de las Administraciones Públicas y de las empresas privadas; con una mención directa al impulso del liderazgo de los profesionales del sector turístico. Normalmente, las referencias a la mejora en la calidad y profesionalización en el ámbito turístico se limitan al ámbito privado, obviando la implicación del sector público; por lo que esta mención resultaba de interés y marcaba una diferencia sustancial que otras propuestas.

Igualmente, el Plan también atendía a la aplicación de los criterios y principios de calidad para el personal que desempeña la atención directa al turista; por cuanto constituye la imagen última del destino Andalucía en los visitantes. Las poblaciones que acogen cada año a los turistas fueron las grandes olvidadas de este Plan, y era en este objetivo dedicado a las personas donde se tendrían que haber incluido las necesarias campañas de sensibilización a los habitantes de las localidades turísticas.

Las actuaciones sobre el producto turístico constituyen el último de los bloques en los que se estructuraba el Plan. Los avances en la calidad de los productos se desarrollaban a partir de un diagnóstico previo de la situación turística andaluza; donde además de valorar los criterios de calidad vigentes, se hacían previsiones de futuro sobre las demandas y expectativas del sector. De esta forma, se disponía de los elementos de juicio necesarios para diseñar los productos turísticos que permitieran elaborar una oferta de calidad. 
El Plan de Calidad tomaba como referencia un conjunto de principios rectores en torno a los cuales se estructuran sus estrategias, programas y actuaciones. Sin perjuicio de los principios que se encuentran previstos en el PGTS, podemos destacar la necesidad de dar visibilidad a la calidad. Cabe recordar que estamos analizando el primero de los instrumentos de planeamiento que regula esta materia en la Comunidad Autónoma de Andalucía; por tanto, uno de los principios de mayor interés es esta proyección de la calidad a todos los sujetos implicados en el sector turístico. La visibilidad de la calidad queda patente en la aplicación de las estrategias, que vamos a analizar bajo la perspectiva de este principio rector.

Las estrategias de este Plan se centraban en cuatro ámbitos de actuación: cooperación multilateral, mejora de los servicios, extensión de las garantías y alineación con el mercado. Destacamos la cooperación y coordinación interadministrativa e intersectoral por cuanto suponen la implicación, e indirectamente la difusión, a todos los niveles administrativos y a todos los sectores. Igualmente, corresponde hacer referencia a la estrategia de mejora de los servicios; que, si bien se centraba en la formación del personal, abría la vía para que otras perspectivas de mejorar de calidad fueran atendidas.

La estrategia de la extensión de las garantías tomaba como punto de partida la necesidad de implantar los estándares adecuados de calidad, a lo largo de todas las fases que comprenden el sector turístico. Esta extensión incidía en la participación en materia de calidad del sector privado. Es evidente que los estándares de calidad se encuentran en constante evolución y están llamados a adaptarse a las demandas de los visitantes, a las novedades tecnológicas y a la evolución natural del mercado; a este respecto, las certificaciones de calidad suponen un apoyo para la extensión de las garantías (Fraguell I Sansbelló, 2011).

En la misma línea, la estrategia dedicada a la alineación con el mercado, como creador de tendencias, ya incluía en este plan una mención a la madurez del sector. Los potenciales visitantes se identificaban ya con un turista que viajaba con regularidad, que comparaba destinos, precios, experiencias, etc., y que comenzaba a marcar los niveles mínimos de calidad que habían de ser ofertados por el sector.

Las estrategias se configuraban como el primer nivel de planificación, concretados a través de los ejes de actuación y de sus programas y actuaciones. Los cuatro ejes de actuación (temático, operativo, de mercado y de planificación), no se identificaban con una estrategia en concreto, si no que en cada uno de ellos confluían una o varias estrategias o algunos de los objetivos y principios.

\subsection{Marco normativo vigente: La Ley $13 / 2011$}

La vigente Ley del Turismo de Andalucía incluye a la calidad como una de sus finalidades y la menciona junto a la profesionalización de los recursos humanos, la innovación y la sostenibilidad, como instrumentos para la mejora de la competitividad del sector turístico. Asimismo, y entre las competencias que corresponden a la Junta de Andalucía en materia turística, en desarrollo de las previsiones estatutarias, menciona la Ley andaluza el fomento "de aquellas medidas y actuaciones que posibiliten el desarrollo y la implantación de políticas de calidad turística en los destinos, recursos, servicios y empresas turísticas de Andalucía” (arts. 1 y 3 ).

Además de estas previsiones generales, la norma andaluza dedica un capítulo específico a la calidad, centrada en la estrategia de actuación que ha de desarrollar la Administración de la Junta de Andalucía. La norma andaluza no define el concepto de calidad, si bien establece unas pautas para la actuación de la administración autonómica, que pueden ayudarnos a identificar cuáles son sus elementos identitarios. Por un lado, se hace referencia en la Ley a la "óptima y homogénea atención a las personas usuarias turísticas en su itinerario de consumo", añadiendo las iniciativas orientadas a la satisfacción de las expectativas de los visitantes y su fidelización; todo ello en el marco de la mejora continuada de los servicios y productos que consuman. El concepto de calidad total en el turismo se traduce en una cultura empresarial basada e la excelencia, que comprende un nivel externo y otro interno. En el nivel externo se trata de satisfacer a los clientes en la mayor medida posible, partiendo del indispensable conocimiento de sus necesidades, motivaciones y deseos. Mientras que en el nivel interno de la empresa se aspira a la máxima eficiencia; ofreciendo al cliente productos en buenas condiciones, con elevadas calidades y a un precio competitivo (Álvarez Sousa, 2003).

La política autonómica en materia de calidad se dirige a establecimientos, destinos y servicios turísticos, y la Ley incluye una enumeración, no taxativa, de los principios que han de regular las iniciativas andaluzas en esta materia. Estos principios se centran en la promoción de los modelos y sistemas de gestión de calidad, los programas específicos para la accesibilidad turística, promoción de las certificaciones o distinciones en materia de calidad, e impulso al análisis de las expectativas y niveles de satisfacción del turismo, y la promoción de sistemas de autocontrol para los servicios turísticos. 
Las previsiones legales a este respecto concluyen con un precepto en el que se remite a la elaboración de un plan específico plurianual, adoptado por la Consejería competente en materia turística, como instrumento para desarrollar la estrategia de calidad turística de la Comunidad. La adopción de este plan queda sujeta a las decisiones que en materia de política turística se adopten en la Administración autonómica, puesto que la norma simplemente lo propone como una vía para la planificación de la calidad turística. Las únicas previsiones que incluye la Ley andaluza se limitan a establecer el órgano competente para su adopción, que será la Consejería responsable del ámbito turístico, y también se precisa que su contenido habrá de respetar en todo caso las previsiones del Plan General del Turismo, como instrumento clave de la planificación turística andaluza (Hall, 2008).

Actualmente, el organigrama de la administración turística autonómica refleja la preocupación por la calidad del sector. En este sentido cabe destacar la Dirección General de Calidad, Innovación y Fomento del Turismo, adscrita a la Consejería de Turismo y Deporte, que ostenta competencias sobre planificación, seguimiento y evaluación de la estrategia de calidad turística, gestión y ejecución de las acciones y programas destinados al apoyo y fomento de la calidad, planes de inspección, mejora de las infraestructuras, y fomento de la formación y el perfeccionamiento de los profesionales del sector turístico, entre otras (art. 8 del Decreto 212/2015, de 14 de julio, por el que se aprueba la estructura orgánica de la Consejería de Turismo y Deporte).

\subsection{El Plan de Calidad Turística de Andalucía 2014-2020}

El Plan General de Turismo Sostenible de Andalucía, instrumento marco en el que se desarrolla la planificación de calidad turística en la Comunidad Autónoma, comprende una línea estratégica denominada "Andalucía: destino turístico de excelencia", que establece las directrices básicas sobre la política de calidad turística andaluza. En esta línea sexta del Plan General se integran cuatro programas de actuación que atienden a las siguientes materias: formación para la profesionalización de los recursos humanos, defensa y protección de los derechos de los usuarios turísticos, accesibilidad universal en los destinos turísticos de Andalucía, y apoyo a la calidad y fomento de la sostenibilidad en las infraestructuras turísticas.

Como complemento de estas referencias genéricas a la actuación de la administración autonómica sobre la calidad turística, se adoptó el III Plan de Calidad Turística de Andalucía 2014 - 2020. Su objetivo es contribuir a la implantación de un nuevo modelo de desarrollo turístico basado en la calidad, la accesibilidad y la sostenibilidad, a la vez que se promueve la innovación a la gestión y el apoyo a sectores poco consolidados; todo ello con la finalidad última de conseguir que el destino Andalucía se defina por la diferenciación y el liderazgo a nivel internacional (Torrejón López, 2017).

Las líneas estratégicas se sitúan sobre tres ejes de actuación: excelencia, innovación y formación, y coordinación y diálogo. Desde la perspectiva de la excelencia, el Plan apuesta por su concepción global e integradora; y, de forma más específica, por aquellos proyectos que permiten la implantación de destinos turísticos basados en la calidad y la excelencia, gracias a la apuesta por la accesibilidad, la sostenibilidad y la protección de los consumidores.

En el texto del Plan se reconoce que Andalucía ocupa una posición destacada en los listados de los indicadores de certificación actuales, y se incide en la necesidad de seguir trabajando en esta línea. A este respecto, y dentro del programa "Destinos Excelentes", se prevén actuaciones destinadas a reconocer y visualizar las empresas y espacios certificados a través de acciones de difusión; así como otras iniciativas como las actuaciones que persigan la puesta en valor de normas de calidad turística, la participación en organismos de apoyo a la calidad, y la realización de estudios y análisis sobre la Satisfacción del Turista en la Comunidad. El apoyo de las Administraciones Públicas es determinante en el fomento de los certificados de calidad, principalmente mediante subvenciones y otro tipo de incentivos; iniciativas públicas que han tenido un impacto directo sobre la calidad, imagen y competitividad del sector turístico (Djofack, 2012).

La apuesta por la accesibilidad se concreta en un programa, también incluido en la línea estratégica dedicada a la excelencia, y que parte de un estudio y análisis de la situación del sector turístico en esta materia. Para animar a las empresas y servicios turísticos a la implantación de criterios de accesibilidad se prevén medidas de apoyo, el desarrollo de herramientas para la difusión y conocimiento de buenas prácticas en accesibilidad para las empresas, el fomento del distintivo de Accesibilidad Turística, y acciones de sensibilización.

También se aborda esta problemática desde la normativa turística, apostando por la incorporación de criterios de accesibilidad como elementos de valor. La finalidad es que estos criterios condicionen la 
categoría y clasificación de los establecimientos y servicios turísticos; además de incluir premisas como la eliminación total de barreras arquitectónicas y la atención especializada del cliente con discapacidad.

La accesibilidad como elemento determinante del nivel de calidad del sector turístico es una apuesta clave desde el punto de vista social, y también desde la perspectiva económica. Por un lado, la Administración está obligada a proyectar los servicios turísticos y facilitar el disfrute de su experiencia a todos los sectores poblacionales, y esta es la perspectiva que debe proyectarse desde el Plan de Calidad. Y, de otro lado, las empresas del sector no sólo han de estar obligadas a atender de forma adecuada a estos clientes, sino que han de ser conscientes del potencial de negocio que implica la inversión en accesibilidad (Jurado Almonte, 2014).

A este respecto, consideramos de gran relevancia el análisis conjunto del programa destinado a la calidad con las iniciativas de formación del sector turístico. El turismo es accesible cuando el personal tiene la formación adecuada para atender las necesidades de estos viajeros. Por tanto, las infraestructuras, el diseño y las tecnologías son esenciales; pero la accesibilidad sólo se alcanzará si a todos estos elementos se une la adecuada preparación del personal que atiende al turista (Hernández-Galán, 2017). Asimismo, corresponde incidir en la relación entre sostenibilidad y distintivos de calidad; la consecución de uno de estos distintivos ha de exigir, en todo caso, el cumplimiento de unos estándares de calidad adecuados en materia de sostenibilidad, que permitan abarcar la perspectiva de recursos materiales y la formación del personal.

El programa "Destinos Sostenibles" comprende un conjunto de actuaciones destinadas a fomentar y difundir la sostenibilidad, de ahí la relevancia de partir de la elaboración de un estudio y análisis de la situación del sector turístico andaluz en este ámbito. La finalidad última es incentivar los destinos turísticos sostenibles que favorezcan el desarrollo económico de la Comunidad, a la vez que se preservan los recursos y valores naturales.

Se encuentran propuestas para sectores específicos, como la cooperación con los proyectos nacionales e internacionales que impulsen el reconocimiento y favorezcan la incorporación de criterios medioambientales en el litoral andaluz; que se materializa en una actuación específica denominada "Bandera azul", para el impulso de programas de calidad en el litoral andaluz.

Otras actuaciones específicas se destinan al impulso y difusión de usos y hábitos turísticos sostenibles en espacios naturales protegidos, el fomento del consumo de productos ecológicos y autóctonos como atractivos turísticos, y el apoyo e incentivo a la incorporación de medidas que incrementen la sostenibilidad de establecimientos y empresas turísticas. En cuanto a la normativa turística, también se incluye en este programa una actuación destinada a su adaptación y revisión en relación al cumplimiento de criterios de sostenibilidad.

Desde la perspectiva de la protección de los consumidores, la apuesta por la calidad de las instituciones autonómicas se centra en un modelo de "Destinos Seguros", como elemento identificativo para los destinos andaluces. En esta línea se incide en el desarrollo de los instrumentos ya previstos en la normativa actual, así como la colaboración con organismos públicos y privados dedicados a la protección de los consumidores, la colaboración con la Inspección Turística y la difusión de los derechos de los consumidores para fomentar la participación activa de los turistas en su defensa.

Entre las actuaciones previstas se encuentra el desarrollo normativo para el impulso y creación de la Unidad para la Asistencia al Turista ${ }^{6}$, el desarrollo de un protocolo para la resolución de conflictos destinado al personal de establecimientos turísticos, la habilitación de las oficinas de turismo de la Junta como "centros express" de resolución de conflictos y la formación específica de su personal a este respecto, y la creación de instrumentos para la detección de infracciones frecuentes, entre otras propuestas.

El Plan de Calidad concibe la innovación como un instrumento para generar valor y dar respuesta a las demandas del turista. En este sentido se incide en una doble dimensión: la innovación a través del desarrollo de herramientas para la mejora de la gestión empresarial; y, desde otra perspectiva, la atención a segmentos turísticos innovadores y poco consolidados que permitan responder al nuevo modelo turístico y contrarresten la estacionalidad del sector.

La innovación tecnológica se estructura en torno a las actuaciones de apoyo a Andalucía Lab y al "Centro de Innovación Turística de Andalucía", como instrumentos para incrementar la cualificación del sector empresarial en el ámbito de la innovación, mejorar la competitividad, aumentar la productividad y crear oportunidades en la nueva economía digital.

El programa destinado a los profesionales turísticos centra sus actuaciones en tres niveles formativos: para los actuales profesionales, para los futuros profesionales y para la ciudadanía en general. Las actividades formativas se centran en el apoyo a programas de formación en el ámbito de la calidad para profesionales turísticos. En colaboración con TURESPAÑA, dependiente del Ministerio de Industria, 
Energía y Turismo, se llevarán a cabo actuaciones para la formación de agentes públicos o privados responsables del desarrollo de los proyectos en destino.

En esta actuación parece muy reseñable la referencia a la apuesta por fomentar la cultura del detalle, y la atención al cliente antes, durante y después del viaje, como parte de la apuesta por la calidad. En la misma línea de formación de profesionales actuales se sitúa la apuesta por la evaluación y acreditación de la experiencia profesional; de esta forma, se trata de convalidar la experiencia laboral y que estos trabajadores con larga trayectoria y experiencia laboral puedan obtener una titulación.

Las actuaciones vinculadas a la formación de futuros profesionales se centran en las acciones de colaboración con las Universidades andaluzas para analizar y adaptar la formación superior a las necesidades del mercado turístico y elaborar un programa de cursos de expertos; así como la convocatoria de becas destinadas a la formación de profesionales que realicen trabajos y estudios en materia de gestión, planificación, programación, fomento, promoción, análisis e investigación en el ámbito turístico.

La formación destinada a la ciudadanía se dirige a los estudiantes de primaria y secundaria, a los que se aspira a concienciar sobre la relevancia del turismo en el desarrollo y sostenibilidad de la Comunidad Autónoma; de esta forma se trata de acercar el turismo a los escolares, a la vez que se incide sobre su importancia y se dignifica la labor de los profesionales del sector, incluso despertando vocaciones entre los futuros profesionales turísticos. La sensibilización de la ciudadanía sobre la relevancia de la industria turística es un elemento clave para la consecución de los niveles de calidad a los que aspira este Plan; y, en estas líneas de actuación, incidir sobre el sector poblacional más joven es aún más relevante por la proyección a medio y largo plazo de esta formación temprana.

La línea estratégica de innovación y formación concluye con el programa destinado a la innovación en segmentos turísticos, que apuesta por los subsectores turísticos innovadores, no prioritarios o poco consolidados, abordando la actividad turística desde una nueva perspectiva.

En especial este programa se centra en iniciativas destinadas al turismo gastronómico y al turismo industrial. A este respecto se realizan actuaciones para impulsar los itinerarios y rutas gastronómicas que apuesten por la calidad en los productos alimentarios y en los servicios turísticos. También se apuesta desde las instituciones autonómicas por la fijación de pautas y requisitos para la elaboración de estas rutas e itinerarios, y el apoyo a los entes gestores responsables de proyectos gastronómicos.

La consolidación del turismo gastronómico pasa por la difusión de los productos que lo configuran, de ahí la relevancia de la innovación tecnológica y el desarrollo de herramientas para la consulta de eventos y productos gastronómicos andaluces (López-Guzmán, 2011). En esta misma línea, se sitúa el impulso a la colaboración a nivel interno de los distintos órganos de la Junta de Andalucía que pueden incidir en el turismo gastronómico; destaca la indispensable colaboración con la Consejería con competencias en materia de agricultura y pesca, como principal responsable para el fomento de los productos autóctonos y de calidad.

Desde la perspectiva del turismo industrial, se incide en la colaboración y participación de la Consejería de Turismo en los organismos que contribuyan a potenciar este segmento turístico; en especial, a través de la Red Española de Turismo Industrial. Al igual que para el turismo gastronómico, se incluye una actuación específica destinada a mejorar el conocimiento sobre este modelo turístico y difundir los proyectos que ya están implantados. Estas labores de conocimiento y difusión se realizan en una doble vía, porque además de acercar a la población y a los potenciales visitantes la riqueza industrial de la Comunidad; también se incluyen acciones para dar a conocer al empresariado las posibilidades para complementar su negocio principal a través de este turismo de experiencias.

La última de las líneas es la titulada "Coordinación y diálogo", y comprende un conjunto de actuaciones para la promoción de alianzas y las acciones de coordinación. Se estructura en dos programas de actuación, dedicados respectivamente a la cooperación, participación y consenso, y al impulso y la difusión.

Las actuaciones que comprenden se centran en la colaboración a nivel interno, y a la proyección exterior. Estos objetivos se materializan a través de propuestas de colaboración con proyectos europeos centrados en la calidad, la innovación y la formación, así como acuerdos con entidades representativas de colectivos de personas con discapacidad. También destacan las iniciativas conjuntas con Administraciones competentes en ámbitos con incidencia en el turismo, como el medio ambiente, la ordenación del territorio, o el consumo.

En esta línea de participación e integración de todos los agentes implicados en el sector y de la ciudadanía, se incluyen proyectos para favorecer espacios para el diálogo y el impulso del sector, y proyectos para la mejora de la gestión turística.

El programa de impulso y difusión se centra en mejorar las herramientas que favorezcan un mayor conocimiento sobre el contenido del Plan de Calidad y los proyectos y actuaciones que genere. Esta 
difusión se dirige a toda la ciudadanía y actores implicados, y muy específicamente a las administraciones y organismos colaboradores con el Plan, para los que se prevé la habilitación de espacios y enlaces específicos.

Con un ámbito de aplicación que supera la esfera del Plan de Calidad, nos encontramos con actuaciones de divulgación e información que se centran en el impulso al Centro de Documentación de la Consejería de Turismo y Comercio, como centro gestor del conocimiento turístico al servicio de investigadores, estudiantes, empresariado, y de toda la ciudadanía con interés en esta materia.

Siguiendo el objetivo de ofrecer la máxima claridad, transparencia y difusión de los proyectos autonómicos, se incluye una actuación dirigida a la apertura al público de las bases de datos estadísticos elaborados por la Consejería de Turismo y Comercio, a fin de que pueden adaptarse a las demandas de los solicitantes.

\section{La experiencia de otras Comunidades Autónomas}

\subsection{Cataluña}

Otras Comunidades Autónomas también han incluido menciones a la calidad en su normativa específica sobre el sector turístico. En el caso de la Ley 13/2002, de 21 de junio, de Turismo de Cataluña, desde la Exposición de Motivos se pone de manifiesto que una de las principales materias que preocupan al legislador catalán son los usuarios turísticos y la necesidad de enfatizar sus derechos y de que reciban un trato adecuado y unos servicios de calidad (Jiménez, 2006).

Asimismo, reconoce la norma catalana entre las finalidades que han de guiar la actividad de las administraciones turísticas y de los sujetos turísticos ${ }^{7}$, la especial atención que ha de recibir el turismo de calidad en el conjunto de las medidas para incrementar las corrientes turísticas; la promoción de la calidad y la competitividad de los servicios turísticos; el fomento de la calidad y la vitalidad de los destinos, las denominaciones y las marcas turísticas; la promoción de la modernización del sector turístico, mediante los incentivos oportunos, para conseguir distintivos, sistemas y normas de calidad de los servicios que ofrece; y el impulso a la formación, la calificación y la especialización de los profesionales del sector turístico (art. 3).

En lo que respecta a la planificación, el Plan de Turismo de Cataluña, instrumento de referencia para la planificación turística en la Comunidad, tiene entre sus objetivos el fomento de una oferta turística de calidad y el estímulo de las actuaciones que refuercen la consideración de Cataluña como marca turística global (art. 13). Por ello, en la regulación de su contenido mínimo se establece una mención específica para que el Plan defina las medidas necesarias para la mejora de la calidad y la competitividad turísticas. La atención a la calidad también se aplica a las empresas turísticas, y entre sus obligaciones se encuentra la prestación de los servicios que ofrezcan con la "máxima calidad" (art. 36).

La Comunidad Autónoma de Cataluña ha ido un paso más allá con la creación de un sistema de calidad turística que se define como "un instrumento técnico-jurídico de la Administración turística de la Generalidad de Cataluña puesto a disposición de los actores económicos turísticos que ejercen su actividad en Cataluña y, por extensión, de los usuarios turísticos que demanden la prestación de los servicios turísticos ofrecidos en Cataluña"8.

La finalidad última de este instrumento es el posicionamiento de la Comunidad Autónoma como un destino turístico de alto valor añadido. El sistema se estructura en tres ámbitos funcionales: categorización, especialidad y excelencia, y su ámbito subjetivo de aplicación comprende a las empresas, establecimientos y actividades turísticas y de interés turístico situados en el territorio catalán (Romagosa, 2011).

Especial atención merecen los principios rectores del sistema, por cuanto nos permiten identificar los caracteres esenciales de este modelo (art. 3 de la citada Orden). El primero de los principios es la corresponsabilidad público-privada, que implica la participación de la Administración turística de la Generalidad de Cataluña de un lado, y de las entidades más representativas del sector de otro, para definir, desarrollar y gestionar todas las actividades que comprende el sistema de calidad turística. Por tanto, la propia configuración y diseño del sistema exigen que el sector privado se implique desde la fase inicial, no sólo en la ejecución o aplicación de las políticas públicas, si no en su concreción.

Los principios de libre adhesión y flexibilidad son dos referentes complementarios que permiten definir la apertura del sistema. Por un lado, el carácter voluntario para integrarse en este sistema que implica una decisión de las empresas y establecimientos para formar parte de esta iniciativa pública, en ningún momento una exigencia. Por otro lado, la flexibilidad se predica de la capacidad de adaptación del sistema a las necesidades de cualquiera de los sujetos interesados en su integración. 
La universalidad se traduce en la búsqueda de una homologación y reconocimiento global, a fin de que los turistas sean capaces de identificar con claridad los elementos de garantía, confianza y credibilidad en las propuestas del sector turístico autonómico.

La objetividad y la seguridad jurídica dotan de fiabilidad a un sistema que persigue la búsqueda de la excelencia. A nivel interno, la necesidad de tomar en consideración la valoración y opinión del cliente, y ponerla en relación con criterios reglados o estándares que permitan realizar una valoración objetiva. Desde una perspectiva externa, las acciones de fomento y promoción turísticas vinculadas al sistema se legitiman a través de la aplicación de los principios de libre concurrencia y no discriminación, y en el respeto de los procesos reglamentados.

\subsection{Canarias}

La Ley 7/1995, de Turismo de Canarias, es la norma de referencia para el sector turístico e incluye numerosas menciones a la calidad a lo largo de su articulado. Desde el Preámbulo, se destaca que entre las novedades que incluye esta disposición se encuentran un importante número de derechos del usuario turístico, que hasta la adopción de este texto no se encontraban regulados; como son el derecho a la calidad de los servicios, a la información veraz, a la seguridad, intimidad y tranquilidad, y a formular quejas y reclamaciones.

Uno de los preceptos de la norma regula el denominado "derecho a la calidad de los servicios", haciendo referencia a la calidad y naturaleza de los servicios en proporción directa con la categoría del establecimiento turístico (art. 17). A continuación, se enumeran las obligaciones de la Administración en los espacios de uso público y las empresas en los establecimientos que gestionan, para garantizar un nivel de limpieza adecuado al uso turístico.

Entendemos que el título del precepto, "derecho a la calidad de los servicios" hace referencia a un concepto mucho más amplio que el desarrollado. La norma canaria reduce la calidad de los servicios turísticos a una vinculación con la categoría, y a la limpieza; en lo que respecta a este último punto, las referencias son básicas e incluidas en cualquiera de las normas reglamentarias sobre esta materia. Incluir una referencia a la limpieza de las papeleras o servicios sanitarios y otros elementos de limpieza pública resulta por completo irrelevante en una norma específica para el sector turístico. Quizás pudiera entenderse la mención al cierre de las parcelas sin edificar y otros espacios con elementos arquitectónicos o naturales, atendiendo a la mejora en la imagen del espacio turístico; al igual que la referencia a la permanencia por espacio superior de sesenta minutos al aire libre. Pero, a pesar de su interés, son indicaciones más propias de otro tipo de norma en cuanto a su rango, así como en cuanto a la materia.

Entre las obligaciones de las empresas turísticas se encuentra el mantenimiento de la calidad de sus servicios; además de recordar expresamente el texto que estas empresas se encuentran obligadas al cumplimiento de las normas y medidas destinadas a potenciar la calidad de los servicios.

En las Directrices de Ordenación del Turismo de Canarias, también se hace referencia a la calidad como uno de los criterios clave para su elaboración; en concreto, se incluye una mención específica a la primacía de la calidad sobre el crecimiento cuantitativo ${ }^{9}$. El nuevo modelo de desarrollo turístico al que aspiran estas Directrices toma como referencia la necesidad de un cambio en los modos de producción y gestión sobre la base de la renovación de la oferta turística existente y el incremento de la competitividad a través del aumento de la calidad y la diversificación de sus productos. También se hace mención a la calidad en relación a la dimensión territorial del modelo turístico, con especial atención al uso eficiente del suelo como recurso, y la conservación de los suelos con valores actuales o potenciales; un espacio turístico de calidad exige un entorno urbano y natural de calidad, lo que requiere una regulación integrada que atienda a todas las potencialidades del entorno (Bethencourt Cejas et al, 2005).

Otro de los elementos esenciales en la planificación turística es la perspectiva de la calidad pero desde el punto de vista de la población, la calidad de vida de los habitantes que reciban a los turistas; y la necesidad de que la actividad turística se dirija hacia la mejora del bienestar social y la conservación de los signos de identidad de cada localidad, integrando el modelo turístico en el entorno económico, cultural, social y ambiental. Esta proyección de la calidad hacia los ciudadanos de los entornos turísticos merece una valoración muy positiva por cuanto supone una visión más amplia del concepto de calidad en el modelo turístico. Si bien son numerosas las planificaciones turísticas que atienden a las peculiaridades de la población local y a la necesidad de integrarlas y hacerlas partícipes del desarrollo turístico de su entorno; incluir las referencias a la calidad y la satisfacción de los residentes, supera esa concepción tradicional de la calidad como un modelo orientado al consumidor del servicio. 
Sería de interés ir un paso más allá, incluyendo a los propios distintivos de calidad y la posibilidad de que también recibieran la opinión de los habitantes de cada localidad, y uno de los factores determinantes para la concesión de estas distinciones fuera la contribución al desarrollo socioeconómico de esa localidad, la protección de sus valores culturales, históricos o ambientales y la percepción de los residentes. De esta forma, se favorecería la identificación de la ciudadanía con los servicios turísticos que se ofrecen en su territorio. Estas iniciativas son todavía más loables en un modelo turístico que parece cada vez más alejado de la ciudadanía y que, en numerosas ocasiones, se ha centrado sólo en satisfacer las necesidades de los visitantes; el resultado ya se está poniendo de manifiesto en ejemplos a nivel nacional e internacional, con casos extremos como las ciudades de Venecia o Barcelona, donde la oposición de los vecinos a los turistas se está convirtiendo en un problema para la convivencia las zonas con más afluencia de visitantes.

En el archipiélago canario, el órgano clave en el ejercicio de las competencias autonómicas en materia de calidad es la Agencia de Calidad Turística de Canarias ${ }^{10}$. La Agencia está compuesta por representantes de la administración autonómica, así como representantes de las entidades gestoras de los sistemas de calidad implantados en Canarias, y de las asociaciones empresariales de los subsectores turísticos con presencia en los organismos de gestión de los sistemas de calidad o promotores de los mismos.

Este órgano colegiado, adscrito a la Dirección General competente en materia de ordenación turística, es responsable del impulso de la cualificación y mejora de la gestión del sector turístico. En concreto, la Agencia ha asumido competencias sobre el apoyo a los sistemas de autorregulación de las condiciones de calidad de los servicios ofrecidos por las entidades turísticas; la difusión de la "cultura de la calidad"; el análisis y las propuestas de mejora sobre los sistemas de calidad relacionados con la prestación de servicios turísticos; la promoción de los sistemas de autoevaluación de la calidad; y el apoyo a la participación de las organizaciones empresariales canarias en los organismos estatales, comunitarios e internacionales de normalización y certificación.

\section{Conclusiones}

Las iniciativas públicas en materia de calidad turística han estado marcadas por la competitividad; la calidad se ha configurado como un instrumento diferenciador del destino y una vía para hacer más atractiva la oferta turística. Sin embargo, en esta última etapa se observa como desde los poderes públicos se está abriendo el concepto de calidad hacia una nueva dimensión que comprende la calidad de vida de los residentes en las zonas turísticas, así como la calidad en el empleo, la sostenibilidad ambiental y la accesibilidad. Todos ellos parámetros que también son diferenciadores pero que no sólo atienden a la vertiente económica de la actividad turística, sino que implican una percepción más amplia del sector y lo integran en el desarrollo socioeconómico del territorio.

Esta evolución no es una realidad aislada, puesto que se integra en la perspectiva actual que paulatinamente se está implantando sobre el sector turístico y que exige su vinculación sobre el territorio y su población. Después de una primera etapa meramente económica y extractiva del sector turístico, pasamos por una segunda fase de inversión y consolidación, para después invertir en la recualificación de los destinos saturados y en su reinvención. Desde la perspectiva de la calidad, la etapa actual sería de consagración y expansión de la calidad en los servicios turísticos y en la actividad turística en su conjunto.

La madurez de este sector en las Comunidades Autónomas analizadas muestra cómo es necesario avanzar un paso más allá y garantizar la calidad no sólo para el turista sino para las localidades; es decir, las infraestructuras de calidad, el empleo de calidad, la conservación y protección ambiental del espacio turístico son una realidad que no sólo afecta al visitante, sino que día a día compromete la calidad de vida de la población. Entender que calidad de vida de un territorio y calidad del sector turístico han de ser elementos indisolubles supone el reconocimiento y la consagración de un modelo turístico que avanza y se integra en el espacio, y que respeta y ayuda a conservar su cultura, su entorno ambiental, sus tradiciones, e incluso su modo de vida.

La apuesta por la sostenibilidad es una de las vertientes de la calidad turística que más claramente redunda en un beneficio para los locales y visitantes, cualquier persona con necesidades especiales que pueda asistir a museos, restaurantes, o cualquier otro establecimiento de ocio y sea atendido de forma adecuada y no va mermada su posibilidad de disfrute de esta experiencia por las dificultades de acceso o comunicación que se le impongan desde estos servicios, es un ciudadano que recibe el servicio adecuado. Por tanto, la accesibilidad de las zonas turísticas suponen calidad de vida para sus ciudadanos 
y calidad en la asistencia turística para los visitantes. Esta percepción de la calidad es una vía que ha de potenciarse en los próximos años y donde las Administraciones Públicas están llamadas a ocupar una posición esencial en cuanto a su fomento e impulso, desde la propia asistencia por el personal de la Administración y en las instalaciones públicas, hasta su completa implantación en el sector privado y para todos los servicios que presta.

Uno de los elementos que deben ser analizados es cómo se puede proyectar la calidad en el modelo de desarrollo turístico actual, donde cada vez tienen más presencia las iniciativas de turismo colaborativo, que quedan fuera de los modelos tradicionales de ordenación turística. La cuestión sería cómo las Administraciones Públicas pueden actuar sobre la calidad de los servicios turísticos que se están ofertando a través de estas iniciativas que adquieren cada vez más presencia en el sector turístico. Tras el análisis de los instrumentos de planificación vigentes no consideramos que exista una respuesta desde las Administraciones ante estas nuevas necesidades.

\section{Bibliografía}

Álvarez Sousa, A.

2003. Turismo y calidad global. Contribución al desarrollo integral. En Blanquer Criado, D., V Congreso de Turismo, Universidad y Empresa. La calidad integral del turismo. Valencia, Tirant lo Blanch.

Álvarez García, J., Fraiz Brea, J., Del Río Rama, M.

2012. Grado de utilización de las herramientas de calidad en el sector de alojamiento turístico español. Pasos - Revista de Turismo y Patrimonio Cultural 10 (5): 495-510.

Beas Secall, L.

2012. Los planes de excelencia turística en España (1992-2006). Una apuesta por la revitalización de los destinos. Revisión de su implantación e impactos. Scripta Nova. Revista Electrónica de Geografía y Ciencias Sociales, XVI (411): 387-424.

Bethencourt Cejas, M., Díaz Pérez, F., González Morales, M., Sánchez Pérez, J.

2005. La medición de la calidad de los servicios prestados por los destinos turísticos: el caso de la isla de La Palma. Pasos - Revista de Turismo y Patrimonio Cultural 3(2): 265-272.

Castro León, J.

2005. La Calidad como herramienta de gestión del Turismo Cultural. Pasos - Revista de Turismo y Patrimonio Cultural 3(1): 143-148.

Djofack, S.

2012. Análisis del impacto del sistema de calidad ISO 2001 y del Sistema de Calidad Turística Española en empresas y organizaciones turísticas: Un estudio empírico en Baleares, Tesis Doctoral, Universitat de les Illes Balears, 2012 (145).

Fraguell I Sansbelló, R.

2011. Las certificaciones ambientales como nuevos instrumentos de gestión turística. El caso de "The Green Key. En Espacios y destinos turísticos en tiempos de globalización y crisis. XII Coloquio de Geografía del Turismo, Ocio y Recreación, Universidad Carlos III, Madrid.

Fuentes Medina, L., Hernández Estárico, E., y Morini Marrero, S.

2016. Q de calidad y satisfacción del turista en el sector hotelero español. Cuadernos de Turismo 37: 203-226.

Hernández Galán, J.

2017. Observatorio de accesibilidad universal del turismo en España, Fundación ONCE, Madrid.

Hall, C.

2008. Tourism Planning. Policies, processes and relationships, Person Education, Londres, $2^{\mathrm{a}}$ edc.

Hernández Ramírez, J.

2008. La imagen de Andalucía en el turismo, Centro de Estudios Andaluces. Consejería de la Presidencia, Junta de Andalucía, Sevilla.

Jiménez, S., y Prats, L.

2006.El turismo en Cataluña: evolución histórica y retos de futuro. Pasos - Revista de Turismo y Patrimonio Cultural 4(2): 153-174.

Jurado Almonte, J.

2014. El turismo accesible en Andalucía. Un producto turístico emergente. Revista de Estudios Andaluces, 31: $1-34$. 
Larrubia Vargas, R., y Luque Gil, A.

2001. Las estrategias para la excelencia y la calidad turística en los destinos maduros. El caso de la provincia de Málaga. Baética: estudios de arte, geografía e historia 23: 75 - 118.

López-Guzmán, T., y Jesus, M.

2011. Turismo, cultura y gastronomía. Una aproximación a las rutas culinarias. International Conference on Tourism \& Management Studies, Algarve (Portugal).

Mariño Romero, J., Sánchez-Franco, M., Martín-Velicia, F., y Hernández Mogollón, J.

2013. Calidad en el sector turístico. Análisis del Estado del Arte. Turismo e innovación: VI Jornadas de Investigación en Turismo, Universidad de Sevilla, Sevilla: 291-312.

Navarro de Vega, A.

1999. Plan de calidad turística española. Antecedentes, desarrollo y puesta en marcha. Estudios Turísticos, 139: 5-13.

Pérez Guerra, R.

2014. La intervención administrativa en el sector turístico español: La política turística. Revista Aragonesa de Administración Pública, 43: 396-413.

Romagosa, F., Priestley, G., y Llurdés, J.

2011. El turismo en el marco de una estrategia de planificación sostenible general en Cataluña. Boletín de la Asociación de Geógrafos Españoles, 57: 2667-193.

Sanz Domínguez, C.

2003. La calidad del turismo en la legislación turística: en particular el caso de Andalucía. En Blanquer Criado, D. V Congreso de Turismo, Universidad y Empresa. La calidad integral del turismo, Tirant lo Blanch, Valencia.

Torrejón López, J.

2017. La ordenación de la oferta turística. En Fernández Ramos, S., y Pérez Monguió, J. Estudio sobre el Derecho del Turismo de Andalucía, Instituto Andaluz de Administración Pública, Sevilla.

Tudela Aranda, J.

2003. La problemática jurídica de la calidad turística. Revista Aragonesa de Administración Pública, 23: $131-154$.

\section{Notas}

1 Elaborado en el marco del Grupo de Investigación Ref. SEJ174, financiado por la Consejería de Economía y Conocimiento de la Junta de Andalucía.

2 En este sentido cabe citar la Directiva 2006/123/CE del Parlamento Europeo y del Consejo, de 12 de diciembre de 2006, relativa a los servicios en el mercado interior, en su considerando 102, apoya los procesos y distintivos de reconocimiento de la calidad en el servicio, específicamente en el ámbito del turismo: "Con el fin de mejorar la transparencia y de favorecer las apreciaciones basadas en criterios comparables en relación con la calidad de los servicios ofrecidos y prestados a los destinatarios, es importante obligar a que se pueda acceder fácilmente a la información sobre el significado de las etiquetas y otras marcas distintivas de dichos servicios. Esta obligación de transparencia tiene especial importancia en sectores como el turismo, especialmente, la hostelería, en los que está muy extendido el uso de sistemas de clasificación. Así mismo, procede averiguar en qué medida la normalización europea puede ser útil para facilitar la compatibilidad y la calidad de los servicios (...)".

3 Cabe precisar, siguiendo a TUDELA ARANDA, que: "La idea de calidad se encontraba ya presente en los primeros ideólogos de la política turística española. Representativa de esta toma de posición es la obra del Marqués de la Vega Inclán, continuamente atravesada por referencias a la protección del patrimonio cultural, del entorno medioambiental, a la mejora de las infraestructuras o de las instalaciones hoteleras" (Tudela Aranda 2003: 135).

4 Además de las referencias a los instrumentos de planificación a nivel estatal, corresponde una mención normativa; en concreto a la Ley 17/2009, de 23 de noviembre, sobre el libre acceso a las actividades de servicios y a su ejercicio. Esta disposición dedica un Capítulo a la "Política de calidad de los servicios", donde se insta a las Administraciones Públicas a fomentar un elevado nivel de la calidad de los servicios. Y, en particular a fomentar que los prestadores aseguren de forma voluntaria la calidad de los servicios por medio, entre otros, de los siguientes instrumentos: a) La evaluación o certificación de sus actividades por organismos independientes; b) La elaboración de su propia carta de calidad o la participación en cartas o etiquetas de calidad elaboradas por organizaciones empresariales o profesionales a nivel comunitario. Entre otras referencias destinadas a la implicación de las organizaciones de consumidores, y los colegios y organizaciones profesionales, y cámaras de comercio, también hace mención la Ley al fomento de las inspecciones administrativas y controles periódicos, así como el diseño y refuerzo de planes de inspección.

5 La primera de las normas de rango reglamentario que reguló la Oficina de la Calidad del Turismo fue el Decreto 351/2003; posteriormente esta norma fue derogada mediante el Decreto 150/2005, de 21 de junio, por el que se reguló la Oficina de Calidad del Turismo de Andalucía. Esta última disposición estuvo en vigor hasta la adopción de la vigente Ley 13/2011, del Turismo de Andalucía, que expresamente se pronunció sobre la derogación de esta disposición. 
$6 \quad$ En el art. 27 de la LTA se regula la Unidad para la Asistencia al Turista en los siguientes términos: "1.-Con el fin de informar y proteger en sus derechos a las personas usuarias de los servicios turísticos, se crea la Unidad para la Asistencia al Turista; 2. Para la consecución de dicha finalidad desarrollará, entre otras, las siguientes funciones: informar sobre los derechos que asisten a las personas usuarias de servicios turísticos y sobre la forma de presentar quejas y reclamaciones para hacerlos efectivos, intervenir para la solución de conflictos entre las personas usuarias y prestadores de servicios cuando sea requerido para ello por las partes afectadas, informar a los prestadores de servicios turísticos sobre buenas prácticas y mejora de los servicios y trasladar a la inspección turística las denuncias o quejas que pudieran ser constitutivas de infracción administrativa; 3. La Consejería competente en materia de turismo dotará a la Unidad de los medios personales y materiales más adecuados para el desarrollo de sus funciones, asimismo, suscribirá los instrumentos de colaboración con otras entidades públicas o privadas que sean convenientes para el cumplimiento de sus fines. Reglamentariamente se desarrollarán su estructura administrativa y su funcionamiento".

7 La norma define a los sujetos turísticos como "el conjunto de personas físicas o jurídicas que, como prestadoras de servicios, promotoras del desarrollo del sector o de actividades concretas, trabajadoras o usuarias, tienen relación directa con el sector turístico" (art. 2 b) de la Ley de Turismo de Cataluña).

8 Orden EMO/418/2012, de 5 de diciembre, mediante la que se establece la estructuración del sistema catalán de calidad turística.

9 Directriz 3, 6, 7, de la Ley 19/2003, de 14 de abril, por la que se aprueban las Directrices de Ordenación General y las Directrices de Ordenación del Turismo de Canarias.

10 Art. 18 del Decreto 24/2016, de 4 de abril, mediante en el que se aprueba el Reglamento Orgánico de la Consejería de Turismo, Cultura y Deportes de Canarias. 